\title{
A maternal high-fat diet during pregnancy and lactation, in addition to a postnatal high-fat diet, leads to metabolic syndrome with spatial learning and memory deficits: beneficial effects of resveratrol
}

\author{
Shih-Wen Li ${ }^{1,2}$, Hong-Ren $\mathbf{Y u}^{1}$, Jiunn-Ming Sheen ${ }^{1}$, Mao-Meng Tiao ${ }^{1}$, You-Lin Tain ${ }^{1}$, \\ I-Chun Lin ${ }^{1}$, Yu-Ju Lin ${ }^{3}$, Kow-Aung Chang ${ }^{4}$, Ching-Chou Tsai ${ }^{5}$ and Li-Tung Huang ${ }^{1,6}$ \\ ${ }^{1}$ Department of Pediatrics, Kaohsiung Chang Gung Memorial Hospital and Chang Gung University College of Medicine, \\ Kaohsiung, Taiwan \\ ${ }^{2}$ Department of Medical Research, Kaohsiung Chang Gung Memorial Hospital, Kaohsiung, Taiwan \\ ${ }^{3}$ Department of Obstetrics and Gynecology, Kaohsiung Chang Gung Memorial Hospital and Chang Gung University, College \\ of Medicine, Kaohsiung, Taiwan \\ ${ }^{4}$ Anesthesiology, Kaohsiung Chang Gung Memorial Hospital and Chang Gung University College of Medicine, Kaohsiung, Taiwan \\ ${ }^{5}$ Department of Obstetrics and Gynecology, Kaohsiung Chang Gung Memorial Hospital and Chang Gung University, College \\ of Medicine, Kaohsiung, Taiwan \\ ${ }^{6}$ Department of Traditional Medicine, Chang Gung University, Linkow, Taiwan \\ Correspondence to: Li-Tung Huang, email: litung.huang@gmail.com \\ Keywords: maternal high-fat diet/obesity; spatial; resveratrol; postnatal high-fat diet; hippocampus \\ Received: August 16, $2017 \quad$ Accepted: November 17, $2017 \quad$ Published: December 06, 2017 \\ Copyright: Li et al. This is an open-access article distributed under the terms of the Creative Commons Attribution License 3.0 \\ (CC BY 3.0), which permits unrestricted use, distribution, and reproduction in any medium, provided the original author and source \\ are credited.
}

\section{ABSTRACT}

We tested the hypothesis that high-fat diet consumption during pregnancy, lactation, and/or post weaning, altered the expression of molecular mediators involved in hippocampal synaptic efficacy and impaired spatial learning and memory in adulthood. The beneficial effect of resveratrol was assessed. Dams were fed a rat chow diet or a high-fat diet before mating, during pregnancy, and throughout lactation. Offspring were weaned onto either a rat chow or a high-fat diet. Four experimental groups were generated, namely $\mathrm{CC}, \mathrm{HC}, \mathrm{CH}$, and $\mathrm{HH}$ (maternal chow diet or high-fat diet; postnatal chow diet or high-fat diet). A fifth group fed with HH plus resveratrol (HHR) was generated. Morris water maze test was used to evaluate spatial learning and memory. Blood pressure and IPGTT was measured to assess insulin resistance. Dorsal hippocampal expression of certain biochemical molecules, including sirtuin 1, ERK, PPARY, adiponectin, and BDNF were measured. Rats in HH group showed impaired spatial memory, which was partly restored by the administration of resveratrol. Rats in HH group also showed impaired glucose tolerance and increased blood pressure, all of which was rescued by resveratrol administration. Additionally, SIRT1, phospho-ERK1/2, and phospho-PPARY, adiponectin and BDNF were all dysregulated in rats placed in $\mathrm{HH}$ group; administration of resveratrol restored the expression and regulation of these molecules. Overall, our results suggest that maternal high-fat diet during pregnancy and/or lactation sensitizes the offspring to the adverse effects of a subsequent high-fat diet on hippocampal function; however, administration of resveratrol is demonstrated to be beneficial in rescuing these effects. 


\section{INTRODUCTION}

Maternal obesity is a pervasive health issue, with over $30 \%$ of child-bearing age women being categorized as obese [1]; the trend of obesity has continually increased between 2005 and 2014 [2]. In addition, maternal high-fat diet/obesity may predispose the offspring to altered energy balance, cardiovascular dysfunction, neuroinflammation, and obesity [3-7]. Furthermore, a combined maternal high-fat diet and postnatal high-fat diet increases the risk of metabolic syndrome in the offspring [8-10].

Metabolic syndrome refers to a cluster of risk factors including obesity, dyslipidemia, hypertension, and insulin resistance [11]. Metabolic syndrome is known to affect cognition and contributes to the development and progression of Alzheimer's disease [12, 13]. We have previously reported that a combined maternal high-fat diet and a postnatal high-fat diet resulted in obesity and hypertension [14]. However, it remains unclear whether a maternal high-fat diet/obesity could program offspring to develop metabolic syndrome upon exposure to a highfat diet post-weaning. Additionally, the effect of this dual exposure to a high-fat diet on the development of cognitive deficits is yet to be determined.

Resveratrol, a natural polyphenolic compound, is produced by plants in response to environmental stress and is found in red grape skin, peanuts, a variety of berries and medicinal plants. Resveratrol possesses important biological properties, including antioxidant, anti-inflammation and neuroprotective effects $[15,16]$. Resveratrol is also able to enhance hippocampal plasticity and hippocampal neurogenesis [17]. Recently, a number of studies have focused on the neuroprotective effects of resveratrol, including its ability to diminish the toxicity induced by the amyloid beta peptide $[18,19]$ and kainic acid [20], as well as prevent cerebral ischemic damage $[16,21]$. In addition, animal studies have demonstrated the efficacy of resveratrol to reverse learning and memory impairments in aged animals [16, 22].

Thus, the purpose of this study was to investigate the effects of maternal high-fat diet during pregnancy and lactation alone, as well as in combination with a postnatal high-fat diet, on metabolic profiles and spatial learning and memory in adult male offspring. Hippocampal SIRT1, adiponectin, ERK1/2, phospho-insulin receptor substrate-1 (phospho-IRS-1), tumor necrosis factor- $\alpha$ (TNF- $\alpha$ ), p66Shc and BDNF levels were examined. In addition, the effect of resveratrol was assessed.

\section{RESULTS}

\section{Body weights}

Before mating female rats were fed a chow diet or a high-fat diet and weighed weekly starting from conception until 5 weeks post conception (Figure 1A). High-fat diet dams had significantly heavier body weight on gestational day 19 compared to chow fed dams $(353 \pm 10.44$ gm vs. $413.68 \pm 10.86$ gm; $\mathrm{P}<0.01)$. Female rats were separated into a control group and an obesity group. After birth, male offspring were weighed on PND 2 (Figure 1B). The offspring were weaned at 3 weeks of age, and assigned to either the chow diet group or the high-fat diet group; Food was provided ad libitum from weaning to 4 months of age. The male rats were weighed again when they were 4 months old (Figure 1C). Our results show that the male offspring from dams with maternal obesity had decreased birth weight. Analysis of weight taken when offspring were 4 months old, shows a significant main effect of both maternal obesity/high-fat diet $[\mathrm{F}(1,76)=13.449, \mathrm{P}<0.001]$, and a postnatal high-fat diet treatment $[\mathrm{F}(1,76)=554.604$, $\mathrm{P}<0.001]$. There was also a significant interaction effect between maternal obesity/high-fat diet and postnatal highfat diet $[\mathrm{F}(1,76)=11.757, \mathrm{P}=0.001]$. Adult offspring in the $\mathrm{HH}$ group were the heaviest, followed by members of the $\mathrm{CH}$ group. Analysis using the Student's $t$-test showed a significant effect of resveratrol treatment to decrease body weights in animals exposed to both treatments (HH vs. HHR; $\mathrm{P}<0.001$ ).

\section{Biochemistry parameters of dams}

On day 19 of lactation, the biochemistry parameters showed increased levels of AST, ALT, cholesterol, triglyceride and plasma glucose in high-fat diet/obesity rats $(\mathrm{p}<0.05)$, whereas HDL was decreased in highfat diet/obesity rats $(\mathrm{p}<0.05)$ (Table 1$)$. Together with increased gestational body weight, these data indicated that a high-fat diet caused obesity in pregnant dams.

\section{Blood pressure}

The blood pressure of 4-month-old male rats was measured (Figure 1D and 1E). We observed a significant main effect for the postnatal high-fat diet treatment $[F(1,14)=24.514, P<0.001]$, however, there was no significant effect of maternal obesity/high-fat diet on systolic blood pressure. A two-way ANOVA showed no significant interaction effects between maternal obesity/ high-fat diet and a postnatal high-fat diet. This result indicated that a postnatal high-fat diet caused increased systolic blood pressure. Additionally, a Student's $t$-test analysis showed that resveratrol treatment significantly restored systolic blood pressure levels in these animals (HHR vs. HH; $\mathrm{P}=0.006$ ). Similarly, we observed a main effect for the postnatal high-fat diet treatment $[\mathrm{F}(1,14)=5.046, \mathrm{P}=0.041]$, however, maternal obesity/ high-fat diet did not significantly affect diastolic blood pressure. A two-way ANOVA showed no significant interaction between maternal obesity/high-fat diet and postnatal high-fat diet. Further analysis using a Student's $t$-test showed that resveratrol treatment significantly restored diastolic blood pressure (HHR vs. $\mathrm{HH} ; \mathrm{P}=0.009$ ). 
Dams

\begin{tabular}{cc}
$\begin{array}{c}\text { Chow diet } \\
\mathbf{N}=7\end{array}$ & $\begin{array}{c}\text { High-fat diet/obesity } \\
\mathbf{N}=\mathbf{1 2}\end{array}$ \\
\hline $83.6 \pm 4.08$ & $143.0 \pm 12.72^{* *}$ \\
$37.3 \pm 5.53$ & $97.8 \pm 8.3^{* *}$ \\
$63.0 \pm 3.83$ & $96.0 \pm 8.23^{* *}$ \\
$45.2 \pm 7.26$ & $75.9 \pm 8.97^{*}$ \\
$52.7 \pm 3.53$ & $44.5 \pm 3.17^{*}$ \\
$214.5 \pm 7.08$ & $256.1 \pm 12.33^{*}$ \\
\hline
\end{tabular}

Values are means \pm SEM; AST, aspartate aminotransferase; ALT, alanine aminotransferase; HDL, high-density lipoprotein. Data were collected from dams on lactating day $19 .{ }^{*} \mathrm{P}<0.05,{ }^{* *} \mathrm{P}<0.01$.

\section{Intraperitoneal glucose tolerance test (IPGTT)}

The blood glucose levels of rats in the $\mathrm{CH}$ and $\mathrm{HH}$ groups were measured at the 15,30,60 and 120 min mark,
Results indicate that blood glucose levels of rats in the $\mathrm{CH}$ and $\mathrm{HH}$ groups were higher compared with those of the CC group (Figure 2A). However, rats treated with resveratrol had blood glucose levels similar to those of rats
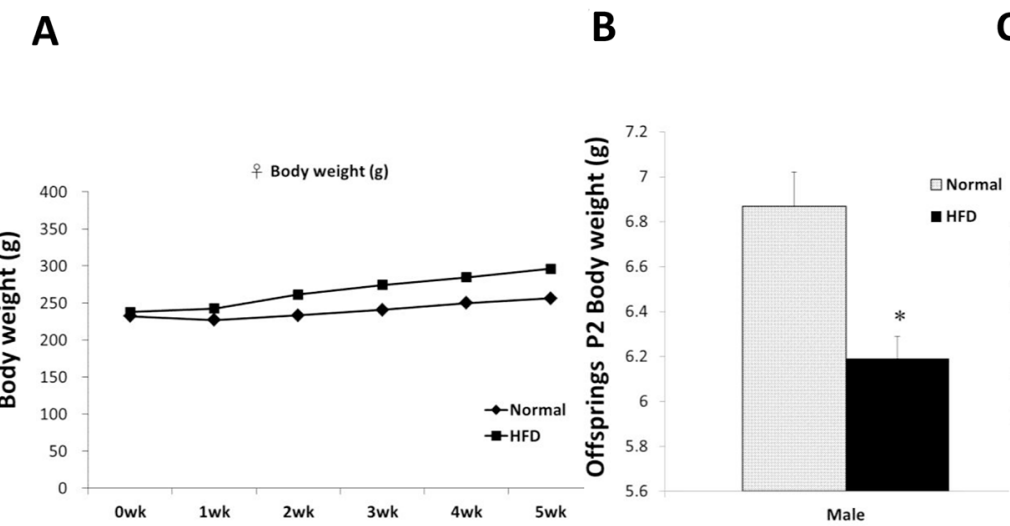

C

D

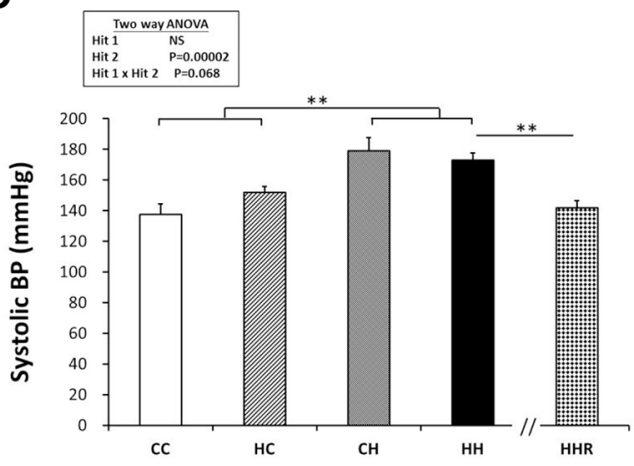

$\mathbf{E}$

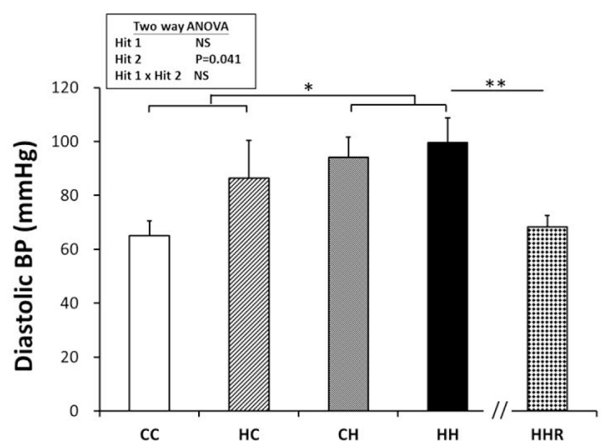

Figure 1: Body weight and blood pressures. (A) Female rats were fed a chow diet or a high-fat diet and weighed weekly for 5 weeks starting from conception. (B) After mating, male pups were weighed on PND 2. The offspring were weaned at 3 weeks of age, and assigned to either the chow diet or high-fat diet ad libitum from weaning up to 4 months of age. (C) The male rats were weighed at 4 months. Body weights were analyzed using two-way ANOVA (maternal diet x post-weaning diet) and the therapeutic effect of resveratrol was evaluated by Student $t$-tests. Hit 1 indicated CC and $\mathrm{CH}$ groups vs. HC and $\mathrm{HH}$ groups. Hit 2 indicated CC and HC groups vs. $\mathrm{CH}$ and $\mathrm{HH}$ groups. (D) Systolic and (E) diastolic blood pressures of 4-month old male rats were analyzed using two-way ANOVA and the therapeutic effect of resveratrol was evaluated by Student $t$-tests. ${ }^{*} \mathrm{P}<0.05 ;{ }^{* *} \mathrm{P}<0.01$. 
in the CC group (Figure 2A). A two-way ANOVA of AUC showed no significant main effect of maternal obesity/ high-fat diet treatment $[\mathrm{F}(1,52)=0.03, \mathrm{P}=0.862]$, however, we observed a significant main effect of postnatal highfat diet treatment $[\mathrm{F}(1,52)=5.76, \mathrm{P}=0.002]$. There was no interaction effect of maternal obesity/high-fat diet and postnatal high-fat diet in $\mathrm{AUC}[\mathrm{F}(1,52)=3.039, \mathrm{P}=0.087]$. A Student's $t$-test showed that resveratrol treatment significantly decreases the AUC (HH vs. HHR; P<0.01). These results indicate that the AUC was larger in the $\mathrm{CH}$ and $\mathrm{HH}$ groups compared to the $\mathrm{CC}$ and $\mathrm{HC}$ groups (Figure 2B), and treatment with resveratrol restored the AUC to levels similar to that observed in controls. Thus, the data shows that exposure to a postnatal high-fat diet results in peripheral insulin resistance and resveratrol treatment is able to reverse this effect.
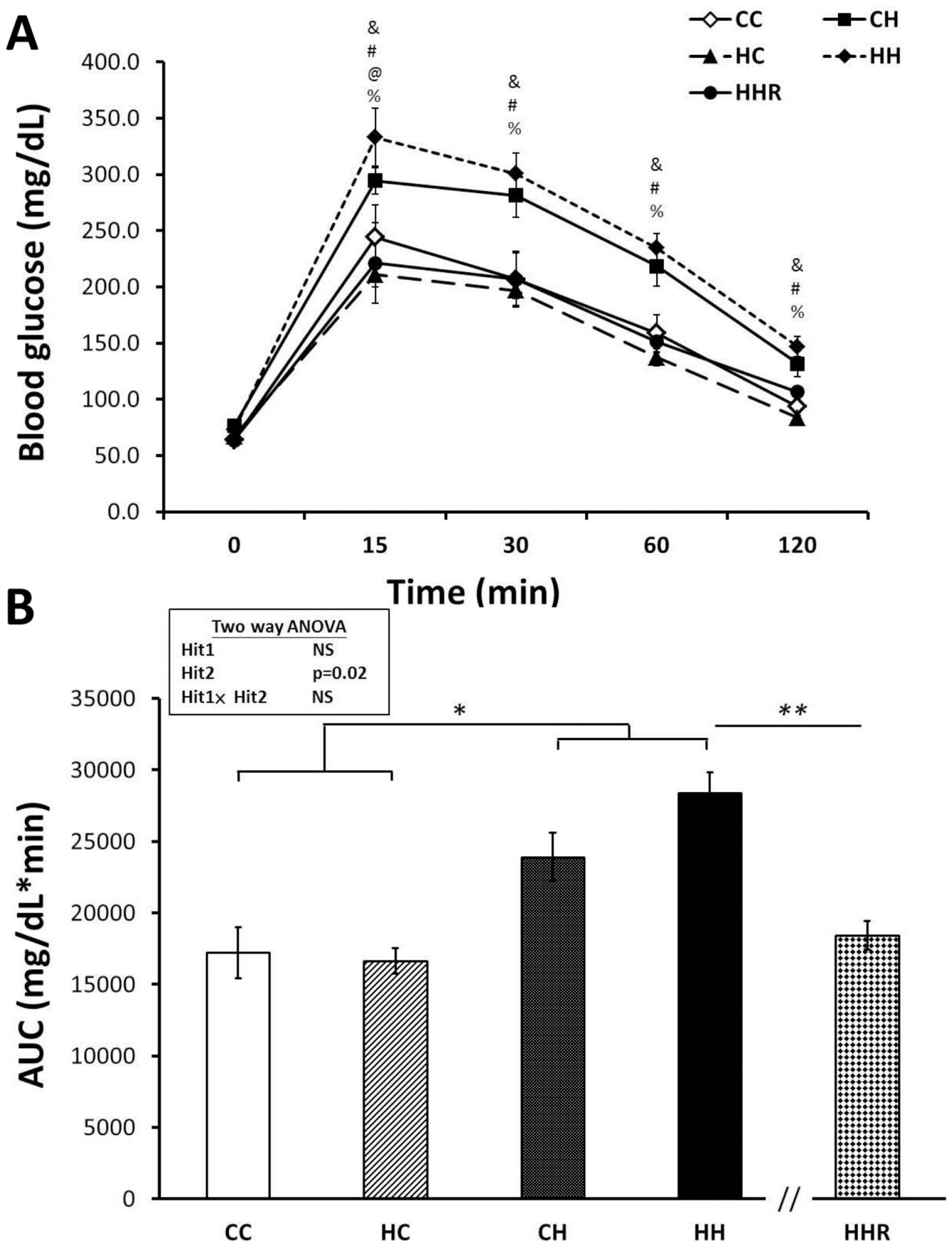

Figure 2: Blood glucose levels of post-stimulation tests. (A) Intraperitoneal glucose tolerance test. (B) Glucose area under the curve (AUC). Results were analyzed using two-way ANOVA (maternal diet x post-weaning diet). \&: $\mathrm{P}<0.05, \mathrm{HH}$ versus $\mathrm{CC}$; \#: $\mathrm{P}<0.05, \mathrm{HH}$ versus HC; ${ }^{@}$ : $\mathrm{P}<0.05$, HH versus $\mathrm{CH} ;{ }^{\%}: \mathrm{P}<0.05$, HH versus HHR; ${ }^{*} \mathrm{P}<0.05 ;{ }^{* *} \mathrm{P}<0.01$. 


\section{Morris water maze}

Acquisition: The water maze tests revealed that all rats were able to learn how to find the platform and that there was no significant difference in swim velocity between the different treatment groups at any time $(\mathrm{P}>0.1)$. Escape latencies improved over time in all four groups as indicated by a significant effect of day of testing on escape latency, indicating that learning occurred (Figure 3). We observed a significant main effect of maternal obesity/high-fat diet on escape latencies $[\mathrm{F}(1,303)=13.829, \quad \mathrm{P}<0.001]$. There was also a significant interaction effect between maternal obesity and postnatal high-fat diet $[\mathrm{F}(1,303)=5.131$,

\section{Morris Water Maze}

A

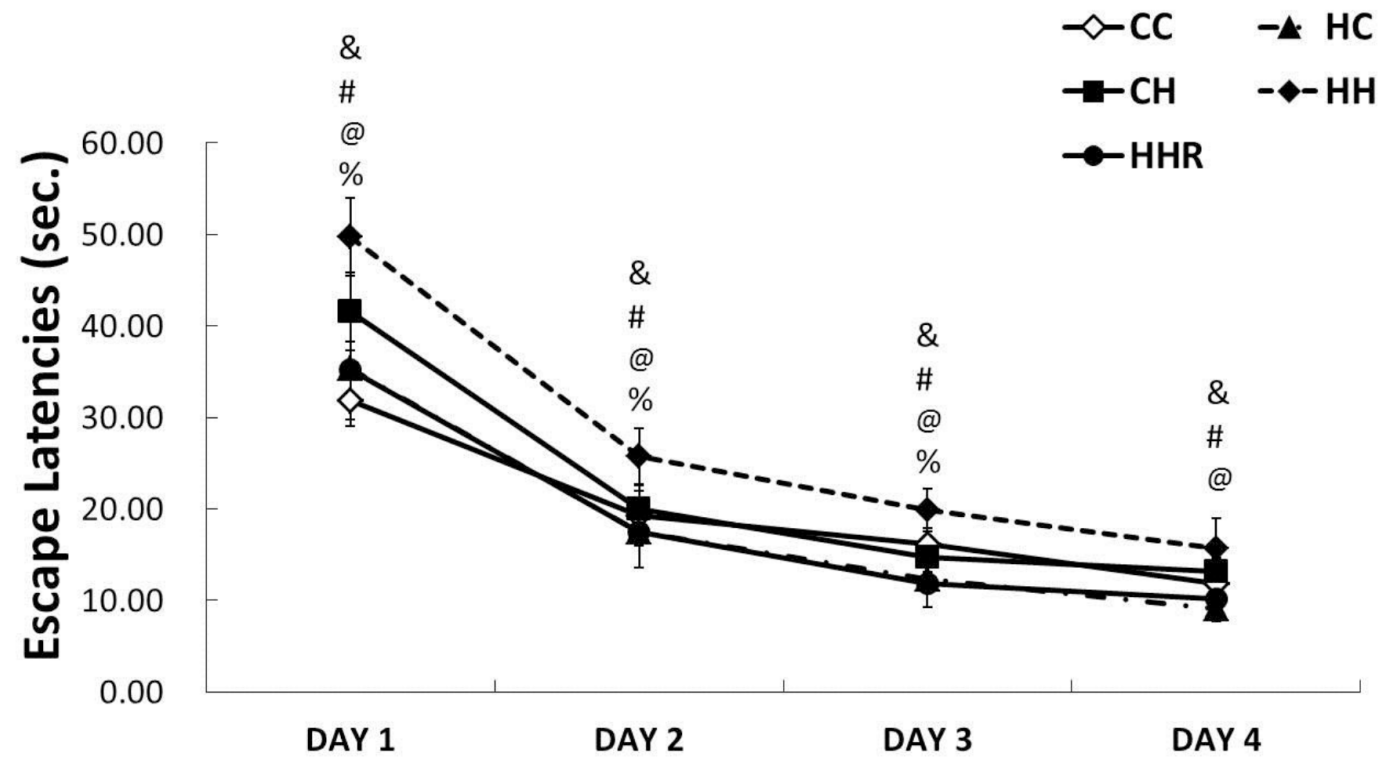

B

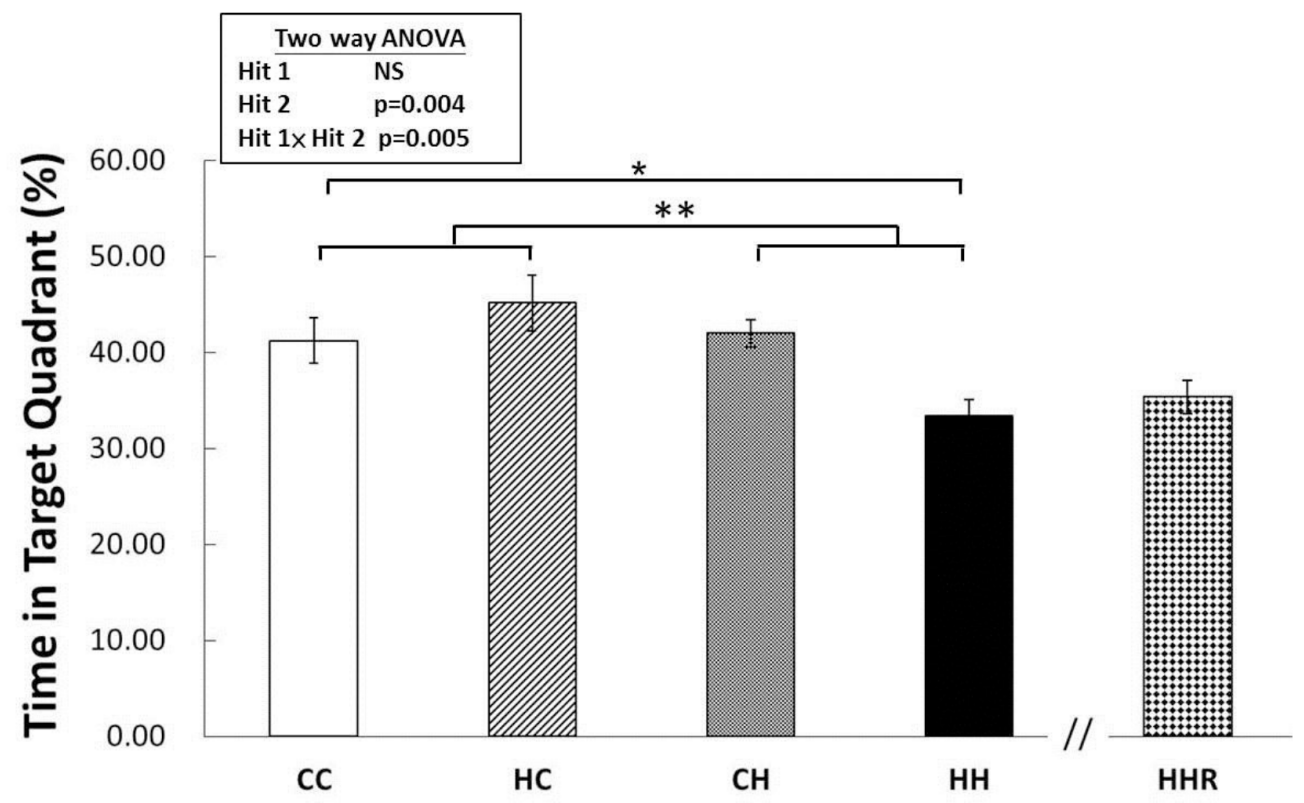

Figure 3: Spatial learning and memory tested using the Morris water maze. (A) Escape latencies to the platform in the Morris water maze (mean $\pm \mathrm{SEM}$ ). Rats in the $\mathrm{HH}$ group swam for a longer period to find the submerged platform on all four acquisition days compared to rats in the $\mathrm{CC}, \mathrm{HC}$, and $\mathrm{CH}$ groups. HHR group rats took less time to reach the platform compared to rats in the HH group. (B) $\mathrm{HH}$ rats on average spent the least amount of time in the target quadrant on day 5 among the four experimental groups. However, there was no significant difference in retention between $\mathrm{HH}$ and HHR rats. A repeated measures ANOVA was used to assess the differences among groups in acquisition. The therapeutic effect of resveratrol was evaluated using post hoc analysis. \&: $\mathrm{P}<0.05, \mathrm{HH}$ versus $\mathrm{CC}$; ${ }^{\#}: \mathrm{P}<0.05, \mathrm{HH}$ versus $\mathrm{HC}$; ${ }^{\circledR}$ : $\mathrm{P}<0.05$, HH versus $\mathrm{CH} ;{ }^{\%}: \mathrm{P}<0.05$, HH versus $\mathrm{HHR} ;{ }^{*} \mathrm{P}<0.05 ;{ }^{* *} \mathrm{p}<0.01$. 
Table 2: Biochemistry parameter of male offspring at 4 months

\begin{tabular}{lccccc}
\hline & $\mathbf{C C}$ & $\mathbf{H C}$ & $\mathbf{C H}$ & HH & HHR \\
& $\mathbf{N = 1 4}$ & $\mathbf{N = 1 4}$ & $\mathbf{N = 1 4}$ & $\mathbf{N = 1 2}$ & $\mathbf{1 4}$ \\
\hline AST (U/L) & $112.92 \pm 6.56$ & $98.28 \pm 6.36$ & $257.7 \pm 24.88^{\& \&}$ & $232.29 \pm 22.87^{\& \&}$ & $200.21 \pm 18.97$ \\
ALT (U/L) & $32.92 \pm 1.04$ & $33.67 \pm 1.69$ & $153.57 \pm 18.72^{\& \&}$ & $113.86 \pm 13.91^{\& \&}$ & $110.5 \pm 15.57$ \\
Cholesterol (mg/dL) & $47.56 \pm 2.19$ & $51.67 \pm 2.82^{\#}$ & $57.48 \pm 2.18^{\& \&}$ & $64.07 \pm 3.43^{\#, \& \&}$ & $50.21 \pm 2.43^{\%}$ \\
Triglyceride (mg/dL) & $83.76 \pm 6.09$ & $91.61 \pm 10.02$ & $75.26 \pm 8.78$ & $101.79 \pm 12.72$ & $64.86 \pm 4.83$ \\
HDL (mg/dL) & $31.28 \pm 2.27$ & $32.72 \pm 2.18$ & $39.3 \pm 1.83^{\& \&}$ & $42.93 \pm 2.4^{\& \&}$ & $32.00 \pm 1.89^{\%}$ \\
Adiponectin (ng/ml) & $1.51 \pm 0.06$ & $1.21 \pm 0.06^{\# \#}$ & $1.2 \pm 0.02^{\& \&}$ & $1.13 \pm 0.04^{\# \#, \& \&}$ & $1.14 \pm 0.04^{\%} \%$ \\
\hline
\end{tabular}

Values are means \pm SEM; AST, aspartate aminotransferase; ALT, alanine aminotransferase; HDL, high-density lipoprotein; $\mathrm{CC}$, maternal control diet + postnatal rat chow normal fat diet; $\mathrm{HC}$, maternal high-fat diet + postnatal rat chow normal fat diet; $\mathrm{CH}$, maternal control diet + postnatal high-fat diet; $\mathrm{HH}$, maternal high-fat diet + postnatal high-fat diet; HHR, maternal high-fat diet + postnatal high-fat diet treat with resveratrol. \#, \#iffers from chow diet treated dams at $\mathrm{P}<0.05$ or 0.01 , respectively, due to a main effect of maternal obesity/high-fat diet treatment. ${ }^{\&}$ \&\& differs from chow diet treated dams at $\mathrm{P}<$ $0.05,0.01$ or 0.001 , respectively, due to a main effect of postnatal high-fat diet treatment. $\%, \%$ : HH vs. HHR evaluated by post hoc test, $\mathrm{P}<0.05$ or 0.01 , respectively.

$\mathrm{P}=0.024]$. Post hoc analysis showed that rats in the $\mathrm{CH}$ group performed better compared to rats in the $\mathrm{HH}$ group $(\mathrm{P}=0.001)$ in the Morris water maze test. Post hoc analysis also showed that resveratrol treatment significantly decreased escape latencies $(\mathrm{P}<0.05)$.

Retention: In the target zone exploration analysis, repeated measures ANOVA showed a significant main effect of postnatal high-fat diet $[\mathrm{F}(1,83)=8.606, \mathrm{P}=0.004]$, as well as a significant interaction between maternal obesity and a postnatal high-fat diet $[\mathrm{F}(1,83)=8.198$, $\mathrm{P}=0.005$ ] in target zone exploration. Post hoc analysis showed that rats in the $\mathrm{CH}$ group performed better in this test compared to rats in the HH group $(\mathrm{P}=0.003)$. This result indicates a retention deficit in $\mathrm{HH}$ rats. However, Student's $t$-test analysis showed that treatment with resveratrol failed to restore the time spent in the target quadrant $(\mathrm{P}=0.411)$.

Overall the data suggest that the combination of maternal obesity/high-fat diet and exposure to a postnatal high-fat diet results in the significant impairment of acquisition and retention in the Morris water maze test. Furthermore, treatment with resveratrol is able to rescue spatial acquisition deficit in $\mathrm{HH}$ group rats.

\section{Biochemistry parameters of male offspring}

The levels of AST, ALT, total cholesterol, and HDL were higher in $\mathrm{HH}$ group rats compared to $\mathrm{CC}$ group rats. However, plasma adiponectin levels were decreased in $\mathrm{HH}$ rats compared to $\mathrm{CC}$ rats (Table 2). Student's $t$-test analysis showed that resveratrol treatment was able to restore total-cholesterol, triglyceride and HDL levels (HH vs. HHR; $\mathrm{p}<0.05)$.

\section{Protein expression levels of adiponectin, phospho-ERK1/2 and phospho-PPAR $\gamma$ in dorsal hippocampus}

Previous studies have associated decreased plasma adiponectin levels with insulin resistance and mild cognitive dysfunction [23-25]. A two-way ANOVA analysis showed a main effect of postnatal high-fat diet treatment $[\mathrm{F}(1,32)=7.285, \mathrm{P}=0.011]$, but not maternal obesity/high-fat $\operatorname{diet}[\mathrm{F}(1,32)=0.538, \mathrm{P}=0.469]$ on hippocampal adiponectin expression. There was also a significant interaction effect between maternal obesity/high-fat diet and a postnatal high-fat diet $[\mathrm{F}(1,32)=6.252, \mathrm{P}=0.018]$. Post hoc analysis showed lower hippocampal adiponectin levels in the $\mathrm{HH}$ group of rats compared to the $\mathrm{CH}$ group of rats $(\mathrm{CH}$ vs. HH: $\mathrm{p}<0.05)$. This result indicates that exposure to a postnatal high-fat diet results in decreased hippocampal adiponectin. It also demonstrates an additive effect of the 2 treatments (Figure 4A and 4B). However, a Student's $t$-test analysis showed that resveratrol treatment failed to restore hippocampal adiponectin expression in HHR group rats as compared with $\mathrm{HH}$ rats $(\mathrm{P}>0.05)$.

Previous studies have shown that PPAR $\gamma$ regulation can be mediated via the ERK1/2 pathway and phosphorylation of PPAR $\gamma$ is involved in insulin sensitivity [26]. A two-way ANOVA showed a significant main effect for postnatal high-fat diet treatment $[\mathrm{F}(1,32)=8.049$, $\mathrm{P}=0.008$ ], but not for maternal obesity/high-fat diet in phospho-PPAR $\gamma$ expression. There was also no significant interaction effect between maternal obesity/high-fat diet and postnatal high-fat diet. This result indicates that exposure to a postnatal high-fat diet results in increased levels of phospho-PPAR $\gamma$ within the dorsal hippocampus (Figure 4A and 4C). Furthermore, Student's $t$-test analysis showed that 
resveratrol treatment did not restore hippocampal phosphoPPAR $\gamma$ levels (HH vs. HHR; $\mathrm{P}>0.05$ ).

A two-way ANOVA revealed a main effect of maternal obesity/high-fat diet treatment $[\mathrm{F}(1,32)=8.522, \mathrm{P}=0.006]$, but not postnatal high-fat diet on phospho-ERK1 expression in the hippocampus. There was no significant interaction effect between maternal obesity/high-fat diet and postnatal high-fat diet. This result indicates that maternal obesity/highfat diet results in a significant increase in hippocampal phospho-ERK1 (Figure 4A and 4D). A Student's $t$-test showed that resveratrol treatment was able to restore hippocampal phospho-ERK1 levels (HH vs. HHR; $\mathrm{P}<0.05)$.

A two-way ANOVA revealed a main effect of maternal obesity/high-fat diet treatment $[\mathrm{F}(1,24)=4.731$, $\mathrm{P}=0.040]$, but not postnatal high-fat diet on hippocampal phospho-ERK2 expression. There was no significant interaction effect between maternal obesity/high-fat diet and a postnatal high-fat diet. This result indicates that maternal obesity results in increased hippocampal phospho-ERK2expression (Figure 4A and 4E). Student's $t$-test showed that resveratrol treatment was able to restore hippocampal phospho-ERK2 levels (HH vs. HHR; $\mathrm{P}<0.05)$.

Overall, we observed that maternal obesity/highfat diet led to an increase in hippocampal phosphoERK1 and -ERK2 expression. On the other hand, a postnatal high-fat diet results in a decreased expression of hippocampal phospho-PPAR $\gamma$. Furthermore, animals subjected to both treatments were observed to have the lowest expression levels of hippocampal adiponectin. Additionally, treatment with resveratrol restored the expression levels of phospho-ERK1 and -ERK2 (HH vs. HHR; $\mathrm{P}<0.05)$, but not adiponectin or phospho-PPAR $\gamma$ (HH vs. HHR; $\mathrm{P}>0.05$ ).

\section{Protein expression levels of phospho- IRS-1, TNF- $\alpha$, SIRT1 and p66Shc in dorsal hippocampus}

In order to determine the effect of maternal obesity/ high-fat diet and a postnatal high-fat diet on central insulin resistance, we assayed for the level of phospho-insulin receptor subunit-1 (IRS-1) in the dorsal hippocampus [27]. A two-way ANOVA showed a main effect of postnatal
A

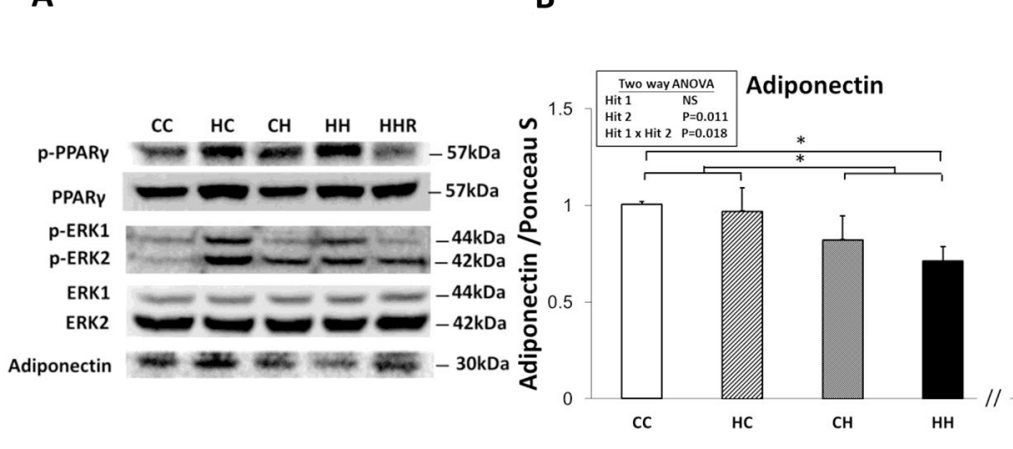

C

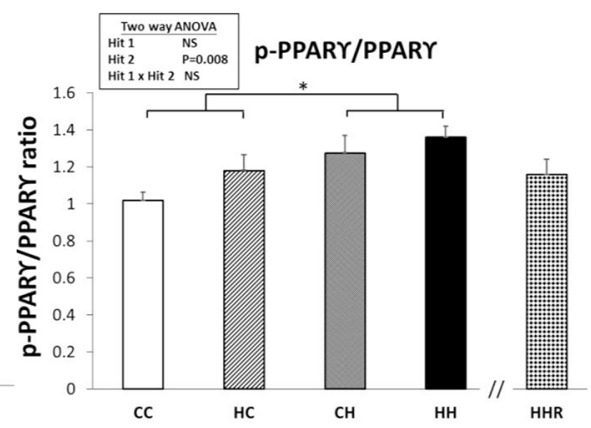

D

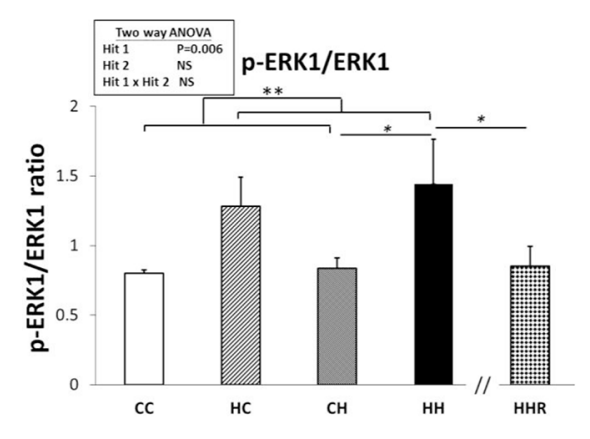

$\mathbf{E}$

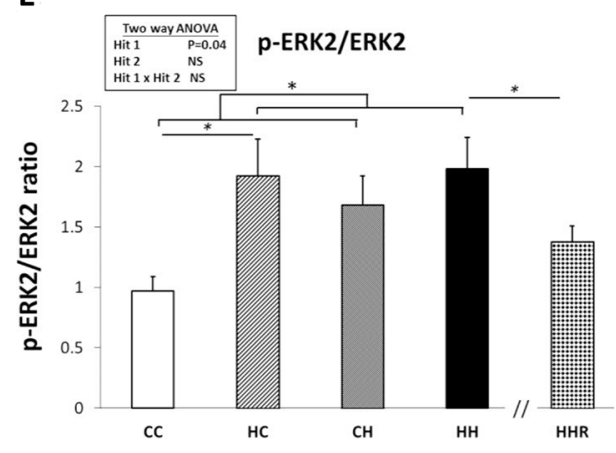

Figure 4: Protein expression levels of adiponectin, phospho-PPAR $\gamma$, phospho-ERK1, and phospho-ERK2 in rat dorsal hippocampus. (A) The expression levels of dorsal hippocampal phospho-PPAR $\gamma$, phospho-ERK1, phospho-ERK2, and adiponectin were detected via western blotting and normalized using Ponceau S staining. Relative abundance of (B) adiponectin, (C) phospho-PPAR $\gamma$, (D) phosphoERK1 and (E) phospho-ERK2 were quantified. A repeated measures ANOVA was used to assess the differences among groups in the expression levels of these molecules and the therapeutic effect of resveratrol was evaluated using the Student $t$-tests. $\mathrm{n}=6$ for each group. ${ }^{*} \mathrm{P}<0.05$; ${ }^{* *} \mathrm{P}<0.01$. 
high-fat diet treatment $[\mathrm{F}(1,29)=6.012, \quad \mathrm{P}=0.020]$, indicating an increase in the expression level of phosphoIRS-1, (i.e., decreased IRS-1 downstream signaling), in the $\mathrm{HH}$ group compared to the CC group (Figure 5A and $5 \mathrm{~B})$. Resveratrol treatment failed to restore the levels of IRS-1 (HH vs. HHR; P>0.05).

To investigate the role of TNF- $\alpha$ in activating the phosphorylation ERK [28], we analyzed the expression level of TNF- $\alpha$ level in the hippocampus (Figure 5A and 5C). A two-way ANOVA showed no interaction effect between maternal obesity/high-fat diet and a postnatal high-fat diet $[\mathrm{F}(1,32)=0.001, \mathrm{P}=0.986]$. However, there was a main effect for postnatal high-fat diet treatment $[\mathrm{F}(1,32)=4.532, \quad \mathrm{P}=0.041], \quad$ indicating that $\mathrm{TNF}-\alpha$ expression levels were higher in $\mathrm{HH}$ group rats compared to $\mathrm{CC}$ group rats. There was, however, no significant main effect of maternal obesity/high-fat diet $[F(1,32)=0.369$, $\mathrm{P}=0.548]$ on hippocampal TNF- $\alpha$ levels. A Student's $t$-test showed that resveratrol treatment did not restore TNF- $\alpha$ expression in the hippocampus (HH vs. HHR; $\mathrm{P}=0.317$ ).

A two-way ANOVA showed a main effect of postnatal high-fat diet treatment on expression levels of SIRT1 $[\mathrm{F}(1,23)=9.030, \mathrm{P}=0.006]$ and of p66Shc $[\mathrm{F}(1,28)=10.341, \mathrm{P}=0.003]$ in the hippocampus. However, there was no effect of maternal obesity/high-fat diet on the expression levels of both SIRT1 and p66Shc in the hippocampus (all $\mathrm{P}>0.05$ ). There was also no significant interaction effect between maternal obesity/high-fat diet and postnatal high-fat diet on the expression level of both proteins in the hippocampus (all $\mathrm{P}>0.05$ ). This result indicates that a postnatal high-fat diet results in decreased hippocampal SIRT1 levels and increased expression of hippocampal p66Shc. A Student's $t$-test showed that resveratrol treatment restored hippocampal SIRT1 and p66Shc expression levels (HH vs. HHR; $\mathrm{P}<0.05$ ).

Together, the above data showed that a postnatal high-fat diet led to increased phospho-IRS-1-S636, TNF- $\alpha$, and p66Shc expression, whereas SIRT1 expression was decreased in the hippocampus. Furthermore, resveratrol treatment restored SIRT1 and p66Shc expression (HH vs. HHR; all $\mathrm{P}<0.05$ ), but not phospho-IRS-1-S636 and TNF- $\alpha$ expression in the hippocampus (HH vs. HHR; all $\mathrm{P}>0.05$ ).

\section{BDNF protein expression levels in dorsal hippocampus}

A two-way ANOVA showed a main effect of postnatal high-fat diet treatment $[\mathrm{F}(1,24)=5.061, \mathrm{P}=0.034]$, but not maternal obesity/high-fat diet on hippocampal BDNF expression (Figure 5A and 5F). This indicates that rats in the $\mathrm{HH}$ group had lower levels of BDNF compared to rats in the $\mathrm{HC}$ group. We also observed an interaction effect between maternal obesity/high-fat diet and postnatal high-fat diet treatments $[\mathrm{F}(1,24)=4.290, \mathrm{P}=0.049]$. Post hoc analysis showed that the $\mathrm{HH}$ group had the lowest $\mathrm{BDNF}$ levels when compared to the $\mathrm{CC}, \mathrm{HC}$, and $\mathrm{CH}$ groups. Student's $t$-test analysis showed that resveratrol treatment restored hippocampal BDNF expression in HHR (HH vs. HHR; $\mathrm{P}<0.05$ ).

\section{DISCUSSION}

We report here that maternal obesity/high-fat diet interacts with a postnatal high-fat diet to induce features of metabolic syndrome, alter biochemical profiles in the dorsal hippocampus, and lead to cognitive deficits. Treatment with resveratrol is able to rescue most of these effects in rats. We have shown that (1) maternal obesity/high-fat diet results in the increased expression of phospho-ERK1 and phospho-ERK2 in the dorsal hippocampus. (2) A postnatal high-fat diet results in increased blood pressure, increased central and peripheral insulin resistance, decreased expression levels of SIRT1, adiponectin, and BDNF, as well as increased expression levels of phospho-PPAR $\gamma$, phospho-IRS1, TNF- $\alpha$, and p66Shc in the dorsal hippocampus. (3) The combination of maternal obesity/high-fat diet and a postnatal high-fat diet led to cognitive impairment and a further decrease in adiponectin and BDNF expression levels in the dorsal hippocampus. (4) Resveratrol treatment restored peripheral insulin resistance, elevated blood pressure, altered dorsal hippocampal phospho-ERK1, -ERK2, SIRT1, p66Shc and BDNF expression to levels observed in control animals, Resveratrol administration was also able to partly rescue cognitive deficits.

An increasing number of studies have demonstrated that offspring exposed to maternal obesity/overnutrition during both pregnancy and lactation are susceptible to increase in adiposity and metabolic dysregulation compared to those from control dams when the offspring themselves are challenged with a high-fat diet after weaning [4, 8, 29-32].

Previous reports showed sex-dependent mechanisms are involved in rat offspring induced by either a maternal high-fat diet and rats fed with a postnatal high-fat diet [14, $33,34]$. Underwood et al. demonstrated that a high-fat diet caused impairment in hippocampus-dependent memory in a sex-dependent manner in rats [35]. In this study, we focused on the cognition and molecular mechanisms in male offspring only. Further studies should be conducted in parallel in both males and females.

In this study, combined maternal obesity/high-fat diet and a postnatal high-fat diet increased body weights, plasma cholesterol and triglycerides. The combined treatment also resulted in increased blood pressure, and impaired glucose tolerance. However, resveratrol treatment restored the increased body weights, elevated blood pressures and impaired glucose tolerance. Taken together, a combined maternal obesity/high-fat diet and a postnatal high-fat diet results in a metabolic syndrome phenotype, and resveratrol treatment is able to rescue this phenotype. 
We observed that maternal obesity/high-fat diet did not result in spatial impairment. This finding was not surprising, given that there have been conflicting results about the effects of maternal obesity on cognitive abilities of offspring. For instance, adult offspring from obese dams have been reported to show normal spatial ability, better spatial performance [32], or impaired spatial performance $[36,37]$ depending on the publication. Animal studies have also reported that rodents maintained on a high-fat diet show impaired learning and cognitive functions [36, 37]. However, normal spatial function following postnatal high-fat diet has also been reported [38-40]. Intriguingly, our study showed that the combined effect of a maternal obesity/high-fat diet and a postnatal high-fat diet led to spatial impairment, and treatment with resveratrol was

A

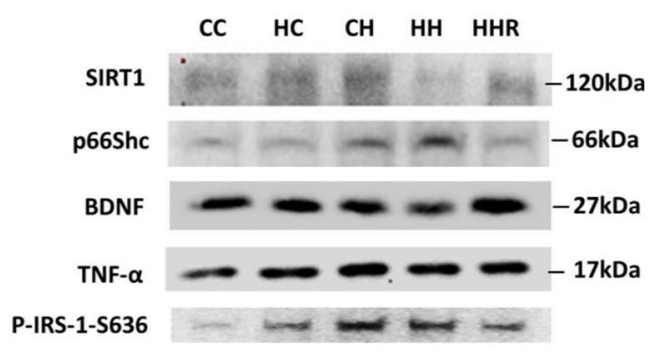

C

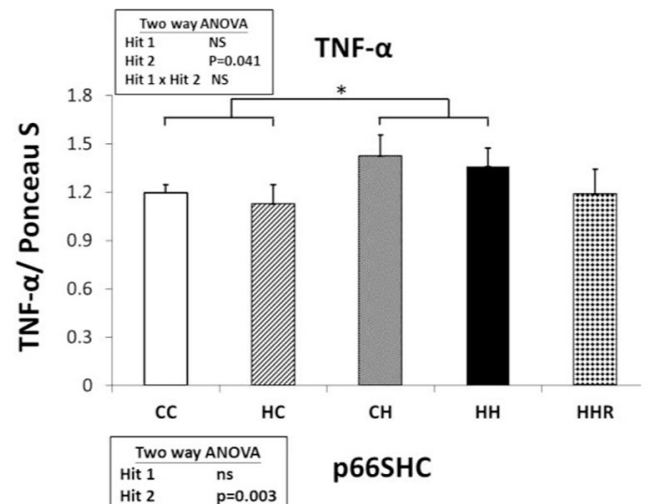

$\mathbf{E}$

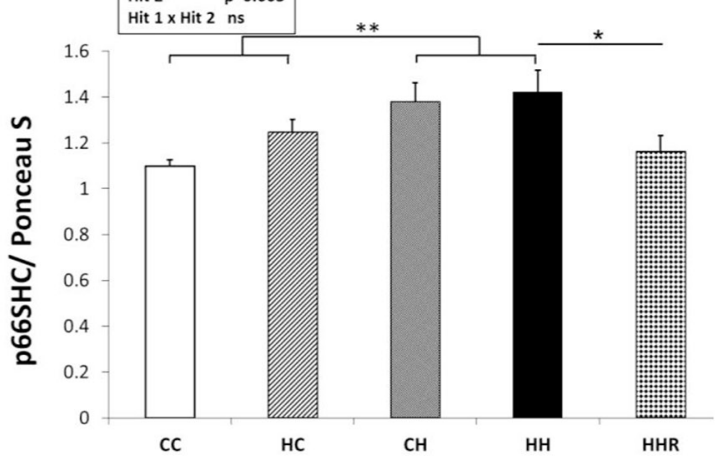

able to reverse the spatial acquisition deficit but not the retention deficit.

Insulin sensitivity is known to be regulated via serine/threonine phosphorylation of IRS-1, in which phospho-IRS-1-Ser636 was associated with desensitization of insulin signaling [27]. Furthermore, activation of the insulin receptor cascade is associated with cognitive function [41]. Insulin resistance in hippocampus is implicated in cognition dysfunction encountered in metabolic syndrome [35, 42-45]. In our study, we demonstrate an upregulation of phospho-IRS1-Ser636 in rats assigned to the $\mathrm{CH}$ and $\mathrm{HH}$ groups. This indicates that a postnatal high-fat diet results in central insulin resistance. However, resveratrol treatment was unable to rescue this effect.

B

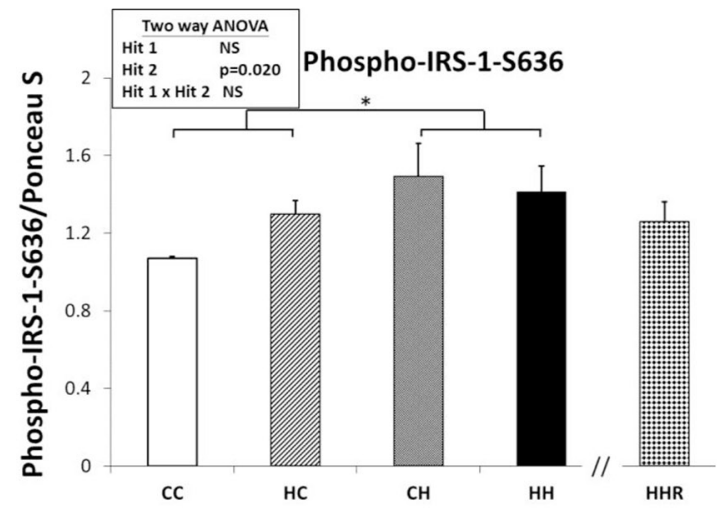

D

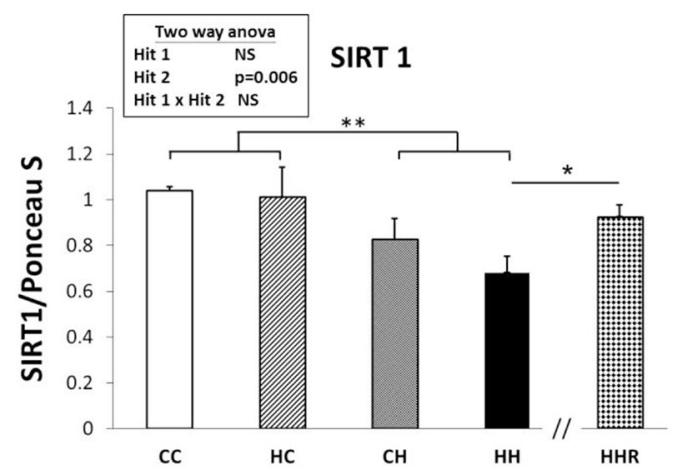

$\mathbf{F}$

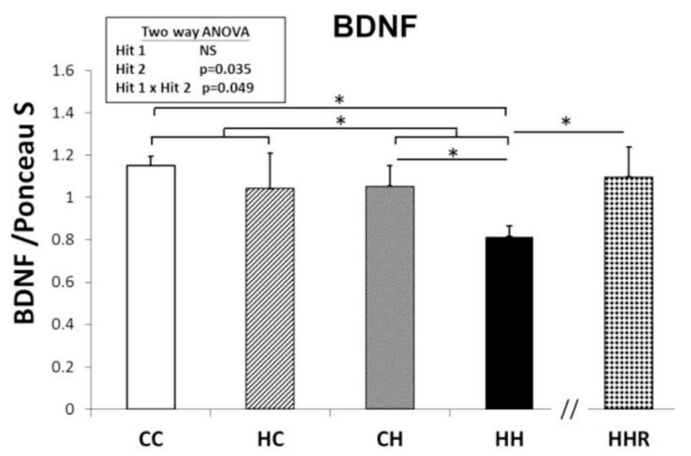

Figure 5: Protein expression levels of phospho-IRS-1-S636, TNF- $\alpha$, SIRT1, p66Shc, and BDNF in rat dorsal hippocampus. (A) The expression levels of IRS-1, TNF- $\alpha$, SIRT1, p66Shc, and BDNF in the dorsal hippocampus were detected via western blotting and normalized using Ponceau S staining. Relative abundance of (B) phospho-IRS-1-S636, (C) TNF- $\alpha$, (D) SIRT1, (E) p66Shc and (F) BDNF were quantified. A repeated measures ANOVA was used to assess the differences among groups and the therapeutic effect of resveratrol was evaluated using the Student $t$-tests. $\mathrm{n}=6$ for each group. ${ }^{*} \mathrm{P} \leq 0.05 ;{ }^{* *} \mathrm{P}<0.01$. 
TNF- $\alpha, \operatorname{PPAR} \gamma$, and ERK are implicated in insulin resistance in metabolic syndrome [45-47]. TNF- $\alpha$, a proinflammatory cytokine, is increased in excessive insulin, obesity and metabolic syndrome [48, 49]. Our study showed that a postnatal high-fat diet led to increased hippocampal expression of TNF- $\alpha$. TNF- $\alpha$ can phosphorylate PPAR $\gamma$ in an ERK-dependent manner in adipose tissue [50], as well as PPAR $\gamma$ can regulate BDNF promoter activity and exert the neuroprotective effects in obese insulin resistant rats $[46,51]$. We observed that maternal obesity/high-fat diet caused increased hippocampal phospho-ERK1 and -ERK2 expression, whereas resveratrol treatment restored them. Our results are consistent with the study conducted by Hosooka et al. that showed that high-fat diet and high glucose incubation increased ERK1/2 phosphorylation as well as phosphorylation of PPAR $\gamma$ [52]. Rats fed a high-fat diet showed decreased adipose tissue adiponectin and increased phosphorylation of ERK and PPAR $\gamma$. These effects were rescued by administration of green tea polyphenols [53]. PPAR $\gamma$ belongs to the PPAR family of nuclear hormone receptors best known for their role in regulating various genes involved in glucose homeostasis and lipid metabolism [24]. In our study, we observed that a postnatal high-fat diet led to increased phosphorylation of PPAR $\gamma$ in rats assigned to the $\mathrm{CH}$ and $\mathrm{HH}$ groups, suggesting a diabetic effect [54].

Adiponectin is also implicated in insulin resistance in metabolic syndrome $[47,55]$. Adiponectin is an adipokine that is inversely correlated with body mass index and fat mass. It induces lipolysis and improves insulin sensitivity in peripheral tissues $[55,56]$. In our study, $\mathrm{HC}, \mathrm{CH}$, and $\mathrm{HH}$ group rats had lower plasma adiponectin as compared with CC group. In parallel, hippocampal adiponectin was decreased in postnatal high-fat rats and was even lower in the $\mathrm{HH}$ group, indicating an additive effect of the dual treatment. However, resveratrol treatment failed to restore decreased plasma or hippocampal adiponectin in rats subjected to both treatments. Previous studies have shown that plasma adiponectin levels are decreased in obesity, insulin resistance and type 2 diabetes [24, 25], Additionally, decreased plasma adiponectin levels have been associated with mild cognitive dysfunction [23]. Hippocampal adiponectin is also known to be involved in cognitive function [39].

Both SIRT1 and p66Shc are implicated in insulin resistance in metabolic syndrome $[57,58]$. A postnatal high-fat diet resulted in decreased expression of hippocampal SIRT1; however, resveratrol treatment was able to restore expression as observed in the HHR

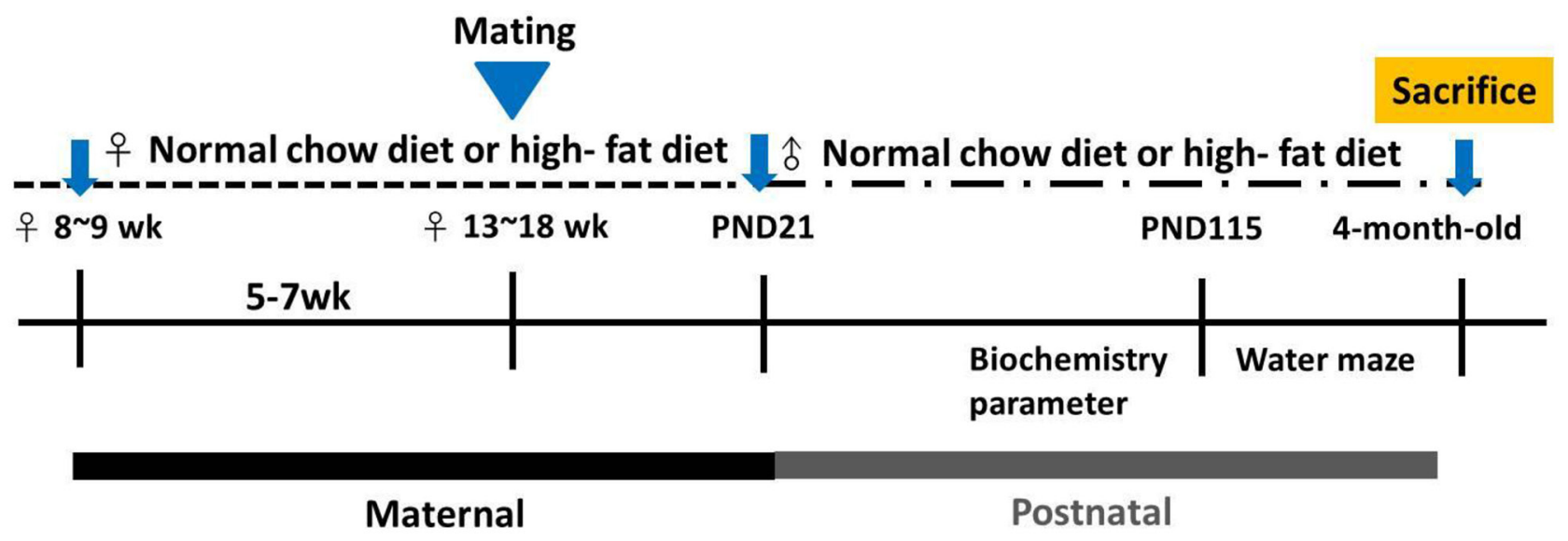

CC: Maternal chow diet + Postnatal chow diet

HC: Maternal obesity/high-fat diet + Postnatal chow diet

CH: Maternal chow diet + Postnatal high-fat diet

HH: Maternal obesity/high-fat diet + Postnatal high-fat diet

HHR: Maternal obesity/high-fat diet + Postnatal high-fat diet + resveratrol

Figure 6: Flowchart of the experimental design. 8-9 weeks old female rats were fed with chow diet or high-fat diet for 5 weeks. After mating and delivery of pups, male baby rats were weaned and fed a chow diet or a high-fat diet from PND21 onwards. Five experimental groups ( $\mathrm{n}=12-14$ per group) were generated: $\mathrm{CC}$ : maternal rat chow diet + postnatal chow diet; HC: maternal obesity/high-fat diet + postnatal chow diet; $\mathrm{CH}$ : maternal rat chow diet + postnatal high-fat diet; $\mathrm{HH}$ : maternal obesity/high-fat diet + postnatal high-fat diet; HHR: maternal obesity/high-fat diet + postnatal high-fat diet + resveratrol. 
group. Mice maintained on a high-fat diet are known to exhibit impaired spatial memory associated with reduced hippocampal SIRT1 mRNA expression [59]. In addition, a high-fat diet may cause insulin resistance, lead to impaired hippocampus-dependent spatial memory and dysregulation of hippocampal SIRT1 via an epigenetic mechanism $[57,60]$. P66Shc mediates obesity-induced insulin resistance [58]. A previous study in human umbilical vein endothelial cells showed that p66Shc was repressed via the binding of SIRT1 to the p66Shc promoter [61]. We have demonstrated that a postnatal high-fat diet leads to increased hippocampal p66Shc in rats assigned to the $\mathrm{CH}$ and $\mathrm{HH}$ groups, and resveratrol treatment is able to restore the levels.

BDNF plays a critical role in hippocampal longterm potentiation, which is a long-term enhancement of synaptic efficacy thought to underlie learning and memory [62]. BDNF is also involved in the pathogenesis of obesity, type 2 diabetes mellitus, and metabolic syndrome $[44,63,64]$. Previous studies have demonstrated that the reduction in BDNF resulting from intake of a highfat diet may impair learning and memory by interfering with hippocampal function [36, 65, 66]. Furthermore, juvenile offspring of obese mothers were reported to have decreased hippocampal BDNF expression and impaired spatial ability; these effects were absent in adult offspring of obese mothers [66]. We observed a lower expression of hippocampal BDNF in rats assigned to the HH group compared to rats assigned to the $\mathrm{CC}, \mathrm{HC}$, and $\mathrm{HH}$ groups, suggesting, an additive effect of maternal high-fat diet/ obesity and a postnatal high-fat diet. Treatment with resveratrol was able to restore the levels of BDNF.

Resveratrol activates SIRT1 and has a variety of biological and pharmacological effects, including cardioprotective, antioxidant, and anti-inflammatory effects [6769]. It is also known to preventing type 2 diabetes mellitus and metabolic syndrome [70]. Resveratrol is also able to increase nitric oxide bioavailability, as well as facilitate the endothelium-dependent vasodilatation necessary for adequate cerebral perfusion [71]. Resveratrol is also able to attenuate hippocampus insulin resistance and spatial deficit in mice induced by a high-fat diet [49]. In addition, resveratrol increased hippocampal SIRT1 activity and preserved hippocampus-dependent memory in mice placed on a high-fat diet [60]. Resveratrol also confers antidepressant-like effects possibly via activation of hippocampal BDNF in rats [72]. In our study, we observe that resveratrol treatment was able to rescue the effects of a high-fat diet on body weight, elevated blood pressure, impaired blood glucose homeostasis and cognitive deficits. Additionally, resveratrol restored expression of phosphoERK1/2, SIRT1, p66Shc, and BDNF in the hippocampus of mice assigned to the HHR group.

In conclusion, exposure to maternal and lactational high-fat diet appears to sensitize the offspring to a postnatal high-fat diet and promotes the development of features related to the metabolic syndrome, including cognitive deficits. In this study, we found altered mediators implicated in insulin resistance and cognition, including adiponectin, PPAR $\gamma$, ERK1/2, p66Shc, SIRT1 and BDNF. Moreover, resveratrol administration is able to reduce the metabolic abnormalities and reverse cognitive deficits in rats exposed to both a maternal and lactational high-fat diet and a postnatal high-fat diet.

\section{MATERIALS AND METHODS}

\section{Animals and experimental design}

This study was carried out in strict accordance with the recommendations of the Guide for the Care and Use of Laboratory Animals of the National Institutes of Health. The protocol was approved by the Institutional Animal Care and Use Committee of the Kaohsiung Chang Gung Memorial Hospital. Virgin Sprague-Dawley (SD) rats (BioLASCO Taiwan Co., Ltd., Taipei, Taiwan) were housed and maintained in a facility accredited by the Association for Assessment and Accreditation of Laboratory Animal Care International. The rats were exposed to a $12 \mathrm{~h}$ light/12 h dark cycle. Male SD rats were caged with female rats until mating was confirmed by examining vaginal plug.

Experimental design was as in Figure 6. Female rats were weight-matched and assigned to receive either a normal diet with regular rat chow (ND; Fwusow Taiwan Co., Ltd., Taichung, Taiwan; 52\% carbohydrates, 23.5\% protein, $4.5 \%$ fat, $10 \%$ ash, and $8 \%$ fiber) or high-fat hypercaloric diet (HF; D12331, Research Diets, Inc., New Brunswick, NJ, USA; 58\% fat [hydrogenated coconut oil] plus high sucrose [ $25 \%$ carbohydrate]) ad libitum for 5 weeks before mating and during gestation and lactation. After birth, litters were culled to 8 pups, with equal numbers of each sex to standardize the received quantity of milk and maternal pup care. The offspring of both sexes were weaned at 3 weeks of age, and onto either the normal diet or high-fat diet ad libitum from weaning to 4 months of age. Five experimental groups $(n=12-14$ per group) were generated: control rat chow diet/postnatal rat chow diet (CC), maternal high-fat diet/postnatal rat chow diet (HC), control rat chow diet/postnatal high-fat diet $(\mathrm{CH})$, and maternal high-fat diet/postnatal high-fat diet [73]. In addition, a therapeutic group with resveratrol on maternal high-fat diet/postnatal high-fat diet was raised for comparison (HHR).

\section{Blood pressure}

Blood pressure was measured in conscious rats at 4 months of age by using an indirect tail-cuff method (BP-2000, Visitech Systems, Inc., Apex, NC, USA) after systematically trained. For each rat, 5 measurements were recorded at each time point as we previously described 
$[14,74]$. Three stable consecutive measures were taken and averaged.

\section{Intraperitoneally injected glucose tolerance test (IPGTT)}

After an 8-h fast at postnatal day (PND) 110, blood samples were collected at 5 time points: before injection and at $15,30,60$, and $120 \mathrm{~min}$ after the intraperitoneally (i.p.) injection of glucose ( $2 \mathrm{~g} / \mathrm{kg}$ body weight). Plasma glucose levels were immediately measured using the enzymatic (hexokinase) method with a glucose assay kit. Serum insulin levels were checked using enzyme-linked immunosorbent assay (Crystal Chem Inc., Downers Grove, IL, USA), as we previously reported [75].

\section{Morris water maze: spatial memory}

The Morris water-maze test was conducted to assess spatial learning and memory in all five groups $[76,77]$. Briefly, at PND 115, each rat was habituated to the training environment. Between PND 116 and 119, the rats were subjected to six trials per day to find the submerged platform. The starting position was changed with each trial. This period was considered as the acquisition phase. At PND $\sim 120$, retention of memory was tested with the platform absent.

\section{Tissue collection and blood sampling}

After lactation, the lactating dams were weighed and euthanized under isoflurane by cervical dislocation ( $n=7-12$ per group). The male offspring were weighed and euthanized at the age of 4 month ( $\mathrm{n}=12-14$ per group). Plasma and dorsal hippocampus were collected. Enzymelinked immunosorbent assay (ELISA) for plasma included triglyceride, liver transaminase, adiponectin, cholesterol, sugar and insulin were according to the manufacturers' protocols.

\section{Western blot}

Western blot analysis was done as described previously [78]. Total protein extracts from homogenized from cultured cells and liver were lysed in ice-cold RIPA buffer with protease inhibitor cocktail (Roche, Indianapolis, IN, USA). After centrifugation, protein concentrations in supernatants were determined by the DC protein assay kit (Bio-Rad, Hercules, CA, USA). The Western blotting technique was performed to quantify the protein density of adiponectin, ERK1/2, BDNF, PPAR $\gamma$ phosphorylation, PPAR $\gamma$ and adiponectin. Briefly, proteins of the left ventricle were isolated, separated by electrophoresis, transferred to a nitrocellulose membrane and probed with one of the following primary antibodies: ERK1/2, BDNF, PPAR phosphorylation, $\operatorname{PPAR} \gamma$ and adiponectin (phosphorylated 1:1000 and total 1:2000). All blots were stained with Ponceau $\mathrm{S}$ as an internal standard, as previously described $[76,79]$. Bands of interest were visualized using electrochemiluminescence reagents (PerkinElmer, Waltham, MA) and quantified by densitometry (Quantity One Analysis software; Bio-Rad), as the integrated optical density (IOD) after subtraction of background. The IOD was baseline adjusted from Ponceau S-staining to correct any variations in total protein loading and an internal standard $[76,80,81]$. The amount of protein was represented as IOD/Std.

\section{ELISA}

In order to detect the adiponectin concentration, we used the rat Adiponectin/Acrp30 DuoSet ELISA (R\&D, DY3100-05) kit to detect the proportional adiponectin concentration from dorsal hippocampus. The adiponectin concentrations were interpolated from standard curve and normalized to total sample mass.

\section{Statistical analysis}

Student's $t$-test was used to compare the body weight and biochemistry parameters between chow and high-fat diet dams. Results were analyzed using twoway analysis of variance (ANOVA) (maternal diet $\mathrm{x}$ postweaning diet), followed by Fisher's LSD post hoc tests if the interaction was significant. For all variables measured, outliers below the first quartile or above the third quartile were removed from the analysis. If there was an additive effect of maternal obesity and postnatal high-fat diet, then the therapeutic effect of resveratrol was evaluated by Fisher's LSD post hoc or unpaired Student $t$-tests. Results from the Morris water maze testing and IPGTT were subjected to repeated measures ANOVA of two-way designed in maternal chowing (normal chow diet vs. high-fat diet), postweaning diet (normal chow diet vs. high-fat diet) were between-subjects factors. The within-subjects factor was days. The major results of probe tests (e.g. dwell time in platform quadrant versus all other quadrants) were compared using one-way ANOVA followed by Fisher's LSD post hoc tests [82]. All analyses were performed using Statistical Package for the Social Sciences (SPSS) software. Values were expressed as mean $\pm \mathrm{SEM}$. Significance was defined as $\mathrm{P}<0.05$ for all tests.

\section{CONFLICTS OF INTEREST}

The authors declare no conflicts of interest.

\section{FUNDING}

This work was supported by grants CMRPG8G0631

(L.T. Huang), CMRPG8F0111 (L.T. Huang), CMRPG8F0141 (H.R. Yu) and CMRPG8F0121 (J.M. 
Sheen) from Chang Gung Memorial Hospital, Kaohsiung, Taiwan and grant MOST 105-2314-B-182-055 (L.T. Huang) from the Ministry of Science and Technology, Taiwan.

\section{REFERENCES}

1. Flegal KM. Metabolically healthy overweight and obesity. Ann Intern Med. 2014; 160:515-6.

2. Flegal KM, Kruszon-Moran D, Carroll MD, Fryar CD, Ogden CL. Trends in obesity among adults in the United States, 2005 to 2014. JAMA. 2016; 315:2284-91.

3. Khan IY, Dekou V, Douglas G, Jensen R, Hanson MA, Poston L, Taylor PD. A high-fat diet during rat pregnancy or suckling induces cardiovascular dysfunction in adult offspring. Am J Physiol Regul Integr Comp Physiol. 2005; 288:R127-33.

4. Bayol SA, Farrington SJ, Stickland NC. A maternal 'junk food' diet in pregnancy and lactation promotes an exacerbated taste for 'junk food' and a greater propensity for obesity in rat offspring. Br J Nutr. 2007; 98:843-51.

5. Chen A, Feresu SA, Fernandez C, Rogan WJ. Maternal obesity and the risk of infant death in the United States. Epidemiology. 2009; 20:74-81

6. Ryckman KK, Berberich SL, Shchelochkov OA, Cook DE, Murray JC. Clinical and environmental influences on metabolic biomarkers collected for newborn screening. Clin Biochem. 2013; 46:133-8.

7. Kang SS, Kurti A, Fair DA, Fryer JD. Dietary intervention rescues maternal obesity induced behavior deficits and neuroinflammation in offspring. J Neuroinflammation. 2014; 11:156

8. Parente LB, Aguila MB, Mandarim-de-Lacerda CA. Deleterious effects of high-fat diet on perinatal and postweaning periods in adult rat offspring. Clin Nutr. 2008; $27: 623-34$.

9. Desai M, Jellyman JK, Han G, Beall M, Lane RH, Ross MG. Maternal obesity and high-fat diet program offspring metabolic syndrome. Am J Obstet Gynecol. 2014; 211:237 e1-13.

10. Ito J, Ishii N, Akihara R, Lee J, Kurahashi T, Homma T, Kawasaki R, Fujii J. A high-fat diet temporarily renders Sod1-deficient mice resistant to an oxidative insult. J Nutr Biochem. 2017; 40:44-52.

11. Grundy SM. Metabolic syndrome update. Trends Cardiovasc Med. 2016; 26:364-73.

12. Yaffe K, Kanaya A, Lindquist K, Simonsick EM, Harris T, Shorr RI, Tylavsky FA, Newman AB. The metabolic syndrome, inflammation, and risk of cognitive decline. JAMA. 2004; 292:2237-42.

13. Yates KF, Sweat V, Yau PL, Turchiano MM, Convit A. Impact of metabolic syndrome on cognition and brain: a selected review of the literature. Arterioscler Thromb Vasc Biol. 2012; 32:2060-7.
14. Tain YL, Lin YJ, Sheen JM, Yu HR, Tiao MM, Chen CC, Tsai CC, Huang LT, Hsu CN. High fat diets sexspecifically affect the renal transcriptome and program obesity, kidney injury, and hypertension in the offspring. Nutrients. 2017.

15. Saiko P, Szakmary A, Jaeger W, Szekeres T. Resveratrol and its analogs: defense against cancer, coronary disease and neurodegenerative maladies or just a fad? Mutat Res. 2008; 658:68-94.

16. Gocmez SS, Gacar N, Utkan T, Gacar G, Scarpace PJ, Tumer N. Protective effects of resveratrol on aging-induced cognitive impairment in rats. Neurobiol Learn Mem. 2016; 131:131-6.

17. Dias GP, Cocks G, do Nascimento Bevilaqua MC, Nardi AE, Thuret S. Resveratrol: a potential hippocampal plasticity enhancer. Oxid Med Cell Longev. 2016; 2016:9651236.

18. Anekonda TS. Resveratrol--a boon for treating Alzheimer's disease? Brain Res Rev. 2006; 52:316-26.

19. Han YS, Zheng WH, Bastianetto S, Chabot JG, Quirion R. Neuroprotective effects of resveratrol against betaamyloid-induced neurotoxicity in rat hippocampal neurons: involvement of protein kinase C. Br J Pharmacol. 2004; 141:997-1005.

20. Zhang LN, Hao L, Wang HY, Su HN, Sun YJ, Yang XY, Che B, Xue J, Gao ZB. Neuroprotective effect of resveratrol against glutamate-induced excitotoxicity. Adv Clin Exp Med. 2015; 24:161-5.

21. Wang Q, Xu J, Rottinghaus GE, Simonyi A, Lubahn D, Sun GY, Sun AY. Resveratrol protects against global cerebral ischemic injury in gerbils. Brain Res. 2002; 958:439-47.

22. Porquet D, Casadesus G, Bayod S, Vicente A, Canudas AM, Vilaplana J, Pelegri C, Sanfeliu C, Camins A, Pallas M, del Valle J. Dietary resveratrol prevents Alzheimer's markers and increases life span in SAMP8. Age (Dordr). 2013; $35: 1851-65$

23. Teixeira AL, Diniz BS, Campos AC, Miranda AS, Rocha NP, Talib LL, Gattaz WF, Forlenza OV. Decreased levels of circulating adiponectin in mild cognitive impairment and Alzheimer's disease. Neuromolecular Med. 2013; $15: 115-21$

24. Kiliaan AJ, Arnoldussen IA, Gustafson DR. Adipokines: a link between obesity and dementia? Lancet Neurol. 2014; 13:913-23.

25. Kadowaki T, Yamauchi T. Adiponectin and adiponectin receptors. Endocr Rev. 2005; 26:439-51.

26. Rangwala SM, Rhoades B, Shapiro JS, Rich AS, Kim JK, Shulman GI, Kaestner KH, Lazar MA. Genetic modulation of PPARgamma phosphorylation regulates insulin sensitivity. Dev Cell. 2003; 5:657-63.

27. Copps KD, White MF. Regulation of insulin sensitivity by serine/threonine phosphorylation of insulin receptor substrate proteins IRS1 and IRS2. Diabetologia. 2012; $55: 2565-82$. 
28. Barbin G, Roisin MP, Zalc B. Tumor necrosis factor alpha activates the phosphorylation of ERK, SAPK/JNK, and P38 kinase in primary cultures of neurons. Neurochem Res. 2001; 26:107-12.

29. Chen LL, Yang WH, Zheng J, Yue L, Hu X. [The effect of high-fat diet and catch-up growth on gastric emptying and plasma glucagon-like peptide- 1 concentration in rats]. [Article in Chinese]. Zhonghua Nei Ke Za Zhi. 2009; 48:655-9.

30. Rajia S, Chen H, Morris MJ. Maternal overnutrition impacts offspring adiposity and brain appetite markers-modulation by postweaning diet. J Neuroendocrinol. 2010; 22:905-14.

31. Torrens C, Ethirajan P, Bruce KD, Cagampang FR, Siow RC, Hanson MA, Byrne CD, Mann GE, Clough GF. Interaction between maternal and offspring diet to impair vascular function and oxidative balance in high fat fed male mice. PLoS One. 2012; 7:e50671.

32. Desai N, Roman A, Rochelson B, Gupta M, Xue X, Chatterjee PK, Tam Tam H, Metz CN. Maternal metformin treatment decreases fetal inflammation in a rat model of obesity and metabolic syndrome. Am J Obstet Gynecol. 2013; 209:136.e1-9.

33. Fu Q, Olson P, Rasmussen D, Keith B, Williamson M, Zhang KK, Xie L. A short-term transition from a high-fat diet to a normal-fat diet before pregnancy exacerbates female mouse offspring obesity. Int J Obes (Lond). 2016; 40:564-72.

34. Tain YL, Lin YJ, Sheen JM, Lin IC, Yu HR, Huang LT, Hsu CN. Resveratrol prevents the combined maternal plus postweaning high-fat-diets-induced hypertension in male offspring. J Nutr Biochem. 2017; 48:120-7.

35. Underwood EL, Thompson LT. A high-fat diet causes impairment in hippocampal memory and sex-dependent alterations in peripheral metabolism. Neural Plast. 2016; 2016:7385314.

36. Molteni R, Barnard RJ, Ying Z, Roberts CK, Gomez-Pinilla F. A high-fat, refined sugar diet reduces hippocampal brain-derived neurotrophic factor, neuronal plasticity, and learning. Neuroscience. 2002; 112:803-14.

37. Andre C, Dinel AL, Ferreira G, Laye S, Castanon N. Dietinduced obesity progressively alters cognition, anxiety-like behavior and lipopolysaccharide-induced depressive-like behavior: focus on brain indoleamine 2,3-dioxygenase activation. Brain Behav Immun. 2014; 41:10-21.

38. Page KC, Jones EK, Anday EK. Maternal and postweaning high-fat diets disturb hippocampal gene expression, learning, and memory function. Am J Physiol Regul Integr Comp Physiol. 2014; 306:R527-37.

39. Pancani T, Anderson KL, Brewer LD, Kadish I, DeMoll C, Landfield PW, Blalock EM, Porter NM, Thibault O. Effect of high-fat diet on metabolic indices, cognition, and neuronal physiology in aging F344 rats. Neurobiol Aging. 2013; 34:1977-87.

40. Hsieh CS, Li SW, Sheen JM, Yu HR, Tiao MM, Tain YL, $\mathrm{Su} \mathrm{CH}$, Huang LT. Postnatal high-fat diet leads to spatial deficit, obesity, and central and peripheral inflammation in prenatal dexamethasone adult offspring rats. Neuroreport. 2016; 27:818-25.

41. Cholerton B, Baker LD, Craft S. Insulin, cognition, and dementia. Eur J Pharmacol. 2013; 719:170-9.

42. Zhang J, Gao Z, Yin J, Quon MJ, Ye J. S6K directly phosphorylates IRS-1 on Ser-270 to promote insulin resistance in response to TNF-(alpha) signaling through IKK2. J Biol Chem. 2008; 283:35375-82.

43. Biessels GJ, Reagan LP. Hippocampal insulin resistance and cognitive dysfunction. Nat Rev Neurosci. 2015; 16:660-71.

44. Wosiski-Kuhn M, Erion JR, Gomez-Sanchez EP, Gomez-Sanchez CE, Stranahan AM. Glucocorticoid receptor activation impairs hippocampal plasticity by suppressing BDNF expression in obese mice. Psychoneuroendocrinology. 2014; 42:165-77.

45. Zhang W, Thompson BJ, Hietakangas V, Cohen SM. MAPK/ERK signaling regulates insulin sensitivity to control glucose metabolism in Drosophila. PLoS Genet. 2011; 7:e1002429.

46. Sripetchwandee J, Pipatpiboon N, Pratchayasakul W, Chattipakorn N, Chattipakorn SC. DPP-4 inhibitor and PPARgamma agonist restore the loss of CA1 dendritic spines in obese insulin-resistant rats. Arch Med Res. 2014; 45:547-52.

47. Kwon H, Pessin JE. Adipokines mediate inflammation and insulin resistance. Front Endocrinol (Lausanne). 2013; 4:71.

48. Moon YS, Kim DH, Song DK. Serum tumor necrosis factoralpha levels and components of the metabolic syndrome in obese adolescents. Metabolism. 2004; 53:863-7.

49. Jeon BT, Jeong EA, Shin HJ, Lee Y, Lee DH, Kim HJ, Kang SS, Cho GJ, Choi WS, Roh GS. Resveratrol attenuates obesity-associated peripheral and central inflammation and improves memory deficit in mice fed a high-fat diet. Diabetes. 2012; 61:1444-54.

50. Tan X, Cao Z, Li M, Xu E, Wang J, Xiao Y. TNF-alpha downregulates CIDEC via MEK/ERK pathway in human adipocytes. Obesity (Silver Spring). 2016; 24:1070-80.

51. Kariharan T, Nanayakkara G, Parameshwaran K, Bagasrawala I, Ahuja M, Abdel-Rahman E, Amin AT, Dhanasekaran M, Suppiramaniam V, Amin RH. Central activation of PPAR-gamma ameliorates diabetes induced cognitive dysfunction and improves BDNF expression. Neurobiol Aging. 2015; 36:1451-61.

52. Hosooka T, Noguchi T, Kotani K, Nakamura T, Sakaue H, Inoue H, Ogawa W, Tobimatsu K, Takazawa K, Sakai M, Matsuki Y, Hiramatsu R, Yasuda T, et al. Dok1 mediates high-fat diet-induced adipocyte hypertrophy and obesity through modulation of PPAR-gamma phosphorylation. Nat Med. 2008; 14:188-93.

53. Brooks NO, Greenstein S, Fry K, Hersh PS. Patient subjective visual function after corneal collagen crosslinking for keratoconus and corneal ectasia. J Cataract Refract Surg. 2012; 38:615-9. 
54. Choi JH, Banks AS, Estall JL, Kajimura S, Bostrom P, Laznik D, Ruas JL, Chalmers MJ, Kamenecka TM, Bluher M, Griffin PR, Spiegelman BM. Anti-diabetic drugs inhibit obesity-linked phosphorylation of PPARgamma by Cdk5. Nature. 2010; 466:451-6.

55. Ruan H, Dong LQ. Adiponectin signaling and function in insulin target tissues. J Mol Cell Biol. 2016; 8:101-9.

56. Yadav A, Jyoti P, Jain SK, Bhattacharjee J. Correlation of adiponectin and leptin with insulin resistance: a pilot study in healthy north Indian population. Indian J Clin Biochem. 2011; 26:193-6.

57. Cao Y, Jiang X, Ma H, Wang Y, Xue P, Liu Y. SIRT1 and insulin resistance. J Diabetes Complications. 2016; 30:178-83.

58. Ranieri SC, Fusco S, Panieri E, Labate V, Mele M, Tesori V, Ferrara AM, Maulucci G, De Spirito M, Martorana GE, Galeotti T, Pani G. Mammalian life-span determinant p66shcA mediates obesity-induced insulin resistance. Proc Natl Acad Sci U S A. 2010; 107:13420-5.

59. Heyward FD, Walton RG, Carle MS, Coleman MA, Garvey WT, Sweatt JD. Adult mice maintained on a high-fat diet exhibit object location memory deficits and reduced hippocampal SIRT1 gene expression. Neurobiol Learn Mem. 2012; 98:25-32.

60. Heyward FD, Gilliam D, Coleman MA, Gavin CF, Wang J, Kaas G, Trieu R, Lewis J, Moulden J, Sweatt JD. Obesity weighs down memory through a mechanism involving the neuroepigenetic dysregulation of Sirt1. J Neurosci. 2016; 36:1324-35.

61. Zhou S, Chen HZ, Wan YZ, Zhang QJ, Wei YS, Huang S, Liu JJ, Lu YB, Zhang ZQ, Yang RF, Zhang R, Cai H, Liu DP, et al. Repression of P66Shc expression by SIRT1 contributes to the prevention of hyperglycemia-induced endothelial dysfunction. Circ Res. 2011; 109:639-48.

62. Cunha C, Brambilla R, Thomas KL. A simple role for BDNF in learning and memory? Front Mol Neurosci. 2010; $3: 1$.

63. Lu B, Nagappan G, Lu Y. BDNF and synaptic plasticity, cognitive function, and dysfunction. Handb Exp Pharmacol. 2014; 220:223-50.

64. Boyuk B, Degirmencioglu S, Atalay H, Guzel S, Acar A, Celebi A, Ekizoglu I, Simsek C. Relationship between levels of brain-derived neurotrophic factor and metabolic parameters in patients with type 2 diabetes mellitus. J Diabetes Res. 2014; 2014:978143.

65. Stranahan AM, Norman ED, Lee K, Cutler RG, Telljohann RS, Egan JM, Mattson MP. Diet-induced insulin resistance impairs hippocampal synaptic plasticity and cognition in middle-aged rats. Hippocampus. 2008; 18:1085-8.

66. Tozuka Y, Kumon M, Wada E, Onodera M, Mochizuki H, Wada K. Maternal obesity impairs hippocampal BDNF production and spatial learning performance in young mouse offspring. Neurochem Int. 2010; 57:235-47.
67. Nie P, Hu W, Zhang T, Yang Y, Hou B, Zou Z. Synergistic induction of erlotinib-mediated apoptosis by resveratrol in human non-small-cell lung cancer cells by down-regulating survivin and up-regulating PUMA. Cell Physiol Biochem. 2015; 35:2255-71.

68. Lin Y, Zhu J, Zhang X, Wang J, Xiao W, Li B, Jin L, Lian J, Zhou L, Liu J. Inhibition of cardiomyocytes hypertrophy by resveratrol is associated with amelioration of endoplasmic reticulum stress. Cell Physiol Biochem. 2016; 39:780-9.

69. Yin K, Zhao L, Feng D, Ma W, Liu Y, Wang Y, Liang J, Yang F, Bi C, Chen H, Li X, Lu Y, Cai B. Resveratrol attenuated low ambient temperature-induced myocardial hypertrophy via inhibiting cardiomyocyte apoptosis. Cell Physiol Biochem. 2015; 35:2451-62.

70. Diaz-Gerevini GT, Repossi G, Dain A, Tarres MC, Das UN, Eynard AR. Beneficial action of resveratrol: how and why? Nutrition. 2016; 32:174-8.

71. Xia N, Forstermann U, Li H. Resveratrol and endothelial nitric oxide. Molecules. 2014; 19:16102-21. https://doi. org/10.3390/molecules191016102.

72. Hurley LL, Akinfiresoye L, Kalejaiye O, Tizabi Y. Antidepressant effects of resveratrol in an animal model of depression. Behav Brain Res. 2014; 268:1-7.

73. Al-Mashham Y, Sinclair BG, Duncan WJ. Giant coronary arterial aneurysms and thrombosis in an infant with Kawasaki disease. Cardiol Young. 2006; 16:510.

74. Tain YL, Sheen JM, Yu HR, Chen CC, Tiao MM, Hsu CN, Lin YJ, Kuo KC, Huang LT. Maternal melatonin therapy rescues prenatal dexamethasone and postnatal high-fat diet induced programmed hypertension in male rat offspring. Front Physiol. 2015; 6:377.

75. Sheen JM, Hsieh CS, Tain YL, Li SW, Yu HR, Chen CC, Tiao MM, Chen YC, Huang LT. Programming effects of prenatal glucocorticoid exposure with a postnatal high-fat diet in diabetes mellitus. Int J Mol Sci. 2016; 17:533.

76. Sheen JM, Chen YC, Hsu MH, Tain YL, Yu HR, Huang LT. Combined intraperitoneal and intrathecal etanercept reduce increased brain tumor necrosis factor-alpha and asymmetric dimethylarginine levels and rescues spatial deficits in young rats after Bile Duct Ligation. Front Cell Neurosci. 2016; 10:167.

77. Kuo CT, Lin YW, Tang NY, Cheng CY, Hsieh CL. Electric stimulation of the ears ameliorated learning and memory impairment in rats with cerebral ischemia-reperfusion injury. Sci Rep. 2016; 6:20381.

78. Li SW, Chen YC, Sheen JM, Hsu MH, Tain YL, Chang KA, Huang LT. Minocycline restores cognitive-relative altered proteins in young bile duct-ligated rat prefrontal cortex. Life Sci. 2017; 180:75-82.

79. Parkhurst CN, Yang G, Ninan I, Savas JN, Yates JR 3rd, Lafaille JJ, Hempstead BL, Littman DR, Gan WB. Microglia promote learning-dependent synapse formation through brain-derived neurotrophic factor. Cell. 2013; 155:1596-609. 
80. Li R, Shen Y. An old method facing a new challenge: re-visiting housekeeping proteins as internal reference control for neuroscience research. Life Sci. 2013; 92:747-51.

81. Tain YL, Hsieh CS, Chen CC, Sheen JM, Lee CT, Huang LT. Melatonin prevents increased asymmetric dimethylarginine in young rats with bile duct ligation. J Pineal Res. 2010; 48:212-21.

82. Remondes M, Schuman EM. Role for a cortical input to hippocampal area CA1 in the consolidation of a long-term memory. Nature. 2004; 431:699-703. 